aspect of patients' life with SLE and their relationship with the healthcare system. Transcripts were analysed through thematic content analysis.

Results The mean age of the participants to the study was 40.6 years $( \pm 10.0)$. The average years since diagnosis were $15.5(S D=8.6)$ with great variability in terms of disease manifestations. Findings showed that a fully engaged patient results from reframing emotional, cognitive, and behavioural dimensions. The advances along the process depends on how the patient succeeds in each position.

Conclusions PHE represents a appropriate model to understand the engagement process of SLE patients. In order to meet patients needs, healthcare providers should consider the specific position of SLE patients, providing adequate and tailored support.

\section{LUPUS EXERCISE AND PERSONAL MEDICATION RECORD ON MOBILE APPLICATION TO HELP IMPROVE THE QUALITY OF LIFE OF PEOPLE WITH LUPUS}

D Syarief. Bandung, Indonesia

10.1136/lupus-2017-000215.149

Background and aims Syamsi Dhuha (Morning Light) Foundation (SDF) has identified two major challenges among the lupus patients who are members of SDF:

- Those who have joint pain and muscle stiffness find it difficult to do their daily activities.

- Those who have to consult two or more doctors/physicians are having difficulties to combine and maintain their medical records in one place

The above challenges brought us to the current works as solutions to the problems

- To create "Lupus Exercise" (LE)

- To create "Lupie Diary Mobile Application" (LD App),

Methods SDF worked together with the Faculty of Sports Education and Health, Education University of Indonesia in developing LE. This LE is recorded and uploaded into YouTube, making it possible for lupus patients to follow the exercise.

SDF worked together with the School of Pharmacy at University of Surabaya, in developing the content for personal medication records. The App is available in Indonesia, English and Mandarin languages, and can be installed on android smartphones.

Results LE is a set of exercise suitable for people living with Lupus, as it:

- can ease joint pain and stiffness if done regularly

- can promote better general health conditions

LD App is a personal medication record application for smartphone users, intended to help lupus patients:

- record their personal medication history

- remind them to take medication and scheduled therapy

- save images of lab test results

Conclusions Both LE and LD App is expected to help people with lupus in coping with Lupus and in helping improve their quality of life.

\section{SLE Complications and comorbidities}

\section{LOW PLASMA CONCENTRATIONS OF APOLIPOPROTEIN M CORRELATE TO DISEASE ACTIVITY AND ENDOTHELIAL DYSFUNCTION IN SLE}

${ }^{1}$ AA Bengtsson* ${ }^{1} \mathrm{H}$ Tydén, ${ }^{1} \mathrm{C}$ Lood, ${ }^{1} \mathrm{~A}$ Jönsen, ${ }^{1} \mathrm{~B}$ Gullstrand, ${ }^{2} \mathrm{~B}$ Dahlbäck. 'Lund University, Clinical Sciences Lund- Rheumatology, Lund, Sweden; 'Lund University-, Laboratory Medicine Malmo- Clinical Chemistry, Malmo, Sweden

\subsection{6/lupus-2017-000215.150}

Background and aims ApoM is an anti-atherogenic and vasculoprotective $25 \mathrm{kDa}$ apolipoprotein suggested to play a role in keeping endothelial barrier integrity. The aims of the current study were to determine the impact of SLE disease activity on apoM levels and investigate if apoM levels reflect endothelial function in SLE.

Methods Plasma concentrations of apoM were measured with ELISA in two SLE cohorts, all patients fulfilling $\geq 4$ American College of Rheumatology (ACR) classification criteria for SLE, and 100 healthy controls (HC). Patients in cohort I had active disease as evaluated with SLEDAI scores. In cohort II endothelial function was measured by EndoPAT 2000 and correlated to apoM levels. A low Reactive Hyperemia Index (RHI) value indicates endothelial dysfunction.

Results In cohort I, the plasma levels of apoM were found to be significantly decreased in SLE $(p<0.0001)$, and the apoM concentrations correlated inversely to disease activity (SLEDAI, $\mathrm{r}=-0.29, \mathrm{p}=0.0063)$. ApoM was also significantly lower in patients with active nephritis, leukopenia, anti-DNA antibodies or rash compared to patients without these manifestations.

In cohort II, using linear regression analysis, there was a positive correlation between apoM levels and the RHI value, indicating endothelial dysfunction, in the younger SLE patients: $\beta=0.94$ CI 95\% 0.22,1.65 $\mathrm{r}=0.32 \mathrm{p}=0.011$.

Conclusions SLE related inflammation may have an impact on lower plasma apoM, which may affect the endothelium and the process towards cardiovascular disease.

\section{HPV-RELATED PREMALIGNANT AND MALIGNANT LESIONS IN THE LOWER GENITAL AND ANAL TRACTS OF WOMEN WITH SLE}

${ }^{1} D$ Dubinsky ${ }^{*},{ }^{1} S$ Sapag Duran, ${ }^{1}$ AM Sapag Duran, ${ }^{1} G$ Nasswetter, ${ }^{2} V$ Maldonado, ${ }^{2} \mathrm{~S}$ Tatti, ${ }^{2} V$ Susuki, ${ }^{3} \mathrm{~L}$ Diaz, ${ }^{3} \mathrm{~L}$ Cardinal, ${ }^{1} \mathrm{AM}$ Beron. ${ }^{1}$ Hospital de Clinicas Jose de San Martin, Reumatologia, Buenos Aires, Argentina; ${ }^{2}$ Hospital de Clinicas Jose de San Martin, Ginecologia, Buenos Aires, Argentina; ${ }^{3}$ Hospital de Clinicas Jose de San Martin, Patologia, Buenos Aires, Argentina

\subsection{6/lupus-2017-000215.151}

Background and Aims Systemic Lupus Erythematosus (SLE) and its treatment predispose to infections such as human papillomavirus (HPV) that is a risk factor for the development of lower genital tract (LGT) and anal cancers.

To assess LGT- anal lesions, frequency of HPV lesions and premalignant and malignant lesions.

Methods Descriptive, cross-sectional design. Women with SLE (ACR 1997) of Argentina were consecutively sent to examination of the LGT and high-resolution anoscopy (2010-2015). Biopsies were performed according to gynaecological criteria and patient consent (Bethesda). Koilocytic cells were associated 